\title{
ORIGINAL RESEARCH \\ Efficiency of Fractional Anisotropy and Apparent Diffusion Coefficient on Diffusion Tensor Imaging in Prognosis of Neonates with Hypoxic-Ischemic Encephalopathy: A Methodologic Prospective Pilot Study
}

O. Brissaud

M. Amirault

F. Villega

O. Periot

J.F. Chateil

M. Allard

\begin{abstract}
BACKGROUND AND PURPOSE: The DTI parameters (FA and ADC) reflect the properties of the brain microstructure. Decreased anisotropy is a common feature of cerebral tissue abnormalities. Our study investigates the neurologic prognostic efficiency of these parameters in white (PLIC, CP) and gray matter (PP) in the first days of life in term neonates with HIE. We hypothesize that lesions in related brain areas could be part of a physiopathologic substratum supporting neurologic deficiencies in this population.
\end{abstract}

\begin{abstract}
MATERIALS AND METHODS: A total of 22 neonates (13 girls and 9 boys; mean gestational age, 40 weeks \pm 9 days; birth weight, $3203 \pm 584 \mathrm{~g}$ ) underwent brain MR imaging between day 1 and day 6 after birth; 6-noncollinear direction DTI was performed. FA and ADC were measured on specific brain areas. Amiel-Tison score was performed on day $8.5 \pm 4$ (group A, favorable outcome $[n=16]$; group $B$, unfavorable outcome $[n=6])$.
\end{abstract}

RESULTS: Intraobserver and interobserver comparison in DTI parameter measurements showed a coefficient of variability of less than $5 \%$. In PLIC and PP, the ADC values were lower in group B compared with group $A(P=.000027)$, whereas in $P L I C$ and $C P$, the FA values were lower in group $B$ compared with group $A(P<.02)$.

CONCLUSIONS: These findings indicate that a poor early neurologic outcome in neonates with HIE is associated with lower FA or ADC values in specific areas of white or gray matter. The difference in $A D C / F A$ changes in the different brain areas explored may support possibly different pathologic processes.

\begin{abstract}
ABBREVIATIONS: $A D C=$ apparent diffusion coefficient; $A S=$ Apgar score at birth $/ 5$ minutes; $\mathrm{BW}=$ birth weight; $\mathrm{CA}=$ cardiac arrest; $\mathrm{CP}=$ cerebral peduncles; $\mathrm{CS}=$ cesarean section; $\mathrm{CV}=$ coefficient of variability; $\mathrm{D}$ = delivery; $\mathrm{DTI}=$ diffusion tensor imaging; $\mathrm{DWI}=$ diffusion-weighted imaging; $E=$ epinephrine; FA, fractional anisotropy; FHRA = fetal heart rate abnormalities; GA = gestational age; $\mathrm{HI}$ = hypoxia-ischemia; $\mathrm{HIE}=$ hypoxic-ischemic encephalopathy; IE = instrumental extraction; IUGR = intrauterine growth retardation; $\mathrm{MRI}=\mathrm{MR}$ imaging; $\mathrm{MSL}=$ meconium-stained liquor; $\mathrm{NI}=$ normal; $\mathrm{NC}=$ nuchal cord; $\mathrm{ND}=$ not done; $\mathrm{PLIC}$, posterior limb of internal capsule; $\mathrm{PP}=$ putamen/pallidum; $\mathrm{S}=$ seizures; $\mathrm{SE}=$ status epilepticus; $\mathrm{SS}=$ Sarnat Score; $\mathrm{T} 1 \mathrm{WI}=$ $\mathrm{T} 1$-weighted imaging; $\mathrm{T} 2 \mathrm{WI}=\mathrm{T} 2$-weighted imaging; $\mathrm{T}=$ thalamus.
\end{abstract}

$\mathbf{S}_{\mathrm{p}}^{\mathrm{s}}$ evere neurologic impairment or death in relationship to perinatal asphyxia in developed countries occurs in 0.2 to 1.3 of 1000 live births. ${ }^{1-6}$ Antenatal and pernatal history, Apgar score, Sarnat classification, Amiel-Tison neurologic assessment, ${ }^{7,8}$ biologic and electrical examinations, and cranial sonography are efficient tools for evaluation of cerebral integrity in neonates. MR imaging, including DWI, contributes to highlighting brain abnormalities. ${ }^{9,10}$ Clinical evaluation and conventional MR imaging parameters are efficient to distinguish severe to minor HIE, but can be inefficient to appreciate long-term outcome for infants with moderate HIE. DTI measures the diffusion of water in cerebral tissue $e^{11-13}$ and quanti-

Received April 24, 2009; accepted after revision June 26.

From Université de Bordeaux, Bordeaux, France.

Please address correspondence to Olivier Brissaud, Unité de Ré animation Pédiatrique et Néonatale, Place Amélie Raba Léon, Hôpital des Enfants, 33076 Bordeaux Cedex, France; e-mail: olivier.brissaud@chu-bordeaux.fr

Indicates article with supplemental on-line tables.

DOI 10.3174/ajnr.A1805 tative parameters such as FA and ADC reflect the microstructure properties in local areas. Decreased anisotropy is a common feature of cerebral tissue abnormalities. ${ }^{13,14}$ Normal and pathologic data for FA and ADC of the neonatal brain structures are available, ${ }^{10,12,13,15-19}$ and some authors suggest that early FA values could be an early prognostic factor for functional outcome in infants with HIE. ${ }^{12}$ The aim of our study was to compare, in full-term neonates suspected to have HIE, the ADC and FA values in specific areas in white matter (PLIC and CP) and in gray matter (PP) with the early neurologic outcome at the end of the first week of life, by using the Amiel-Tison score. ${ }^{7}$ We hypothesized that lesions in related brain areas could be part of a physiopathologic substratum supporting neurologic deficiencies in neonates with HIE.

\section{Materials and Methods}

\section{Patients}

During 1 year, we prospectively enrolled 22 consecutives neonates ( 13 girls, 9 boys; mean gestational age, 40 weeks \pm 9 days; median, 40 
weeks plus 2 days; birth weight $3203 \pm 584 \mathrm{~g}$ ). The inclusion criteria were gestational age older than 37 weeks, an Apgar score at 5 minutes of 5 or less, late deceleration on fetal monitoring, bradycardia lower than $80 \mathrm{bpm}$ at 5 minutes, cardiac arrest, and/or the need for intratracheal or intravenous administration of epinephrine. All neonates with chromosomal disease, cardiac or central nervous system malformations, congenital infection, or inborn metabolic errors were excluded (immediately or secondarily).

The local Human Ethics Committee approved the study, and we obtained parental informed consent.

\section{Clinical Data Collection}

Antenatal history, birth conditions, birth weight, sex, gestational age at birth, Apgar score, Sarnat classification ${ }^{8}$ in the first hours of life, and the day of the MR imaging examination were recorded. Apgar score at 5 minutes was considered good if the score was more than 5 ( 9 infants) and poor if 5 or less (10 infants).

\section{Early Neurologic Outcome}

During the first week of life, or as soon as possible after tracheal tube removal and drug decrease, the neonates underwent neurologic examination (ie, early neurologic outcome) by a pediatrician specializing in neurology (F.V.), blinded to the MR imaging data. We identified 2 groups according to the Amiel-Tison neurologic assessment at term $^{7}$ : no disability, minor disability, or moderate disability (group A) and severe disability or death (group B).

\section{MR Imaging and Postprocessing}

All patients were intubated during MR imaging examination (between day 1 and day 6). All neonates were carried to the MR imaging unit in a transport incubator accompanied by an intensivist experienced in neonatology who remained in attendance throughout the scanning process. No neonate was too unstable to support medical transport. Temperature, blood pressure, heart rate, and all vital signs were monitored during transport. Sedation was adapted to optimize data acquisition (intravenous opioids and/or midazolam). Ventilation was done manually during the MR imaging examinations. Pulse oximetry and electrocardiogram (Maglife, Brucker, France) were monitored throughout the procedure. All infants wore ear protections (Natus MiniMuffs; Natus Medical, San Carlos, California).

We collected all MR imaging data using a 1.5T magnet (Gyroscan Intera system; Philips, Best, the Netherlands) equipped of $20 \mathrm{mT} / \mathrm{m}$ gradients.

MR Imaging Methods. Acquisition was performed with a sensitivity encoding standard head coil, with the following sequences:

- T1-weighted spin-echo sagittal sections (TR, 479 ms; TE, 17 ms);

- T1-weighted inversion-recovery axial sections (TR, $2097 \mathrm{~ms}$; TI, $850 \mathrm{~ms}$; TE, $14 \mathrm{~ms}$ );

- T2-weighted turbo spin-echo axial (TR, $4409 \mathrm{~ms}$; TE, $135 \mathrm{~ms}$ ) and coronal (TR, $4258 \mathrm{~ms}$; TE, $130 \mathrm{~ms}$ ) sections;

- DWI with single-shot echo-planar imaging (TR, $2367 \mathrm{~ms}$; TE, 88 ms; 16 sections; NSA, 2; b-value, $0 / 800 \mathrm{~s} / \mathrm{mm}^{2}$; section thickness, 5 mm; FOV: $250 \times 250$; matrix, $112 \times 90)$; and

- DTI with pulsed field gradients in 16 (6 noncollinear) directions with single-shot echo-planar imaging (TR, $7000 \mathrm{~ms}$; TE, $81 \mathrm{~ms} ; 40$ sections; NSA, 3; b-value, $0 / 700 \mathrm{~s} / \mathrm{mm}^{2}$; section thickness, $2.2 \mathrm{~mm}$; FOV, $176 \times 176$; matrix, $80 \times 80$; DTI time acquisition, 6 minutes; total scanning time $\leq 1$ hour).

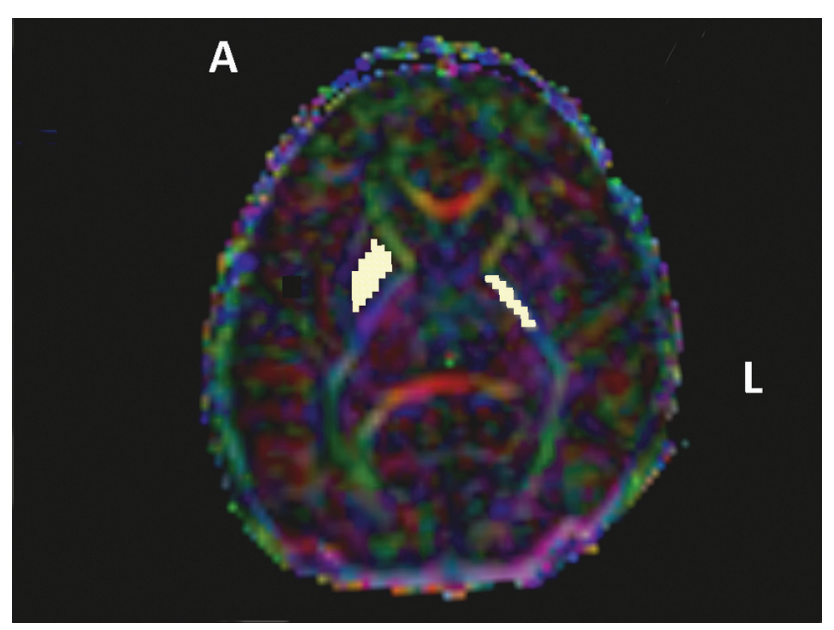

Fig 1. PP and PLIC (white squares) region-of-interest positioning on native tensor diffusion map. The 3 vector elements were assigned to red ( $x$ element, left-right), green ( $y$, anteroposterior), and blue (z, superior-inferior $)$ with a proportional intensity scale according to the FA values.

\section{Postprocessing and MR Imaging Data Analysis}

Conventional MR images and DWI. Images were analyzed by a senior radiologist well experienced in interpreting neonatal brain MR imaging scans (J.F.C.), blinded to clinical ratings and DTI findings and who established an imaging severity score. This score (from $0-22$ ) was generated according to consensual literature reports on MR imaging and DWI abnormalities in neonates with HIE. ${ }^{9,20}$ The higher the MR imaging score, the worse the prognosis for the infants.

DTI postprocessing. DTI data were postprocessed (quantification of the ADC and FA), by use of the PRIDE fiber-tracking tool software (Philips Research Integrated Development Environment, Best, the Netherlands). DTI-based color maps were created from the FA values. Diffusion encoded FA-weighted images were calculated according to the method proposed by Pajevic and Pierpaoli. ${ }^{21}$ For all region-of-interest analyses, voxels were selected from the FA maps in native space, as described previously, ${ }^{22}$ by 2 co-investigators (O.B., M.A.) blinded to the subject's clinical and outcome status, according to anatomic landmarks as follows:

- PLIC: Map at the level of the basal ganglia, where the angle between anterior and PLIC was the most acute (Fig 1).

- PP: At the same section level as PLIC. Voxels were selected on the putamen and the pallidum (Fig 1).

- CP: We first chose on an anatomic map the level where the cerebral peduncles were the most visible. We selected the section located between the superior and inferior colliculi. Voxels were then positioned on the FA map (Fig 2).

FA and ADC measurements by PRIDE were performed for each region of interest at the left and right side. DTI voxel regions were selected in relationship to their greater vulnerability in term neonates with HIE.

\section{Statistical Analyses}

Comparison between qualitative data was performed with the Fisher exact test (Apgar score at 5 minutes vs early neurologic assessment).

Intraobserver and interobserver comparison: Both manual (PLIC, $\mathrm{PP}$ ) and single-point (CP) methods were used for region-of-interest positioning on the FA maps. The size of the region of interest varied according to the individual anatomy of the patients. Interobserver and intraobserver error concerning FA and ADC values were ex- 


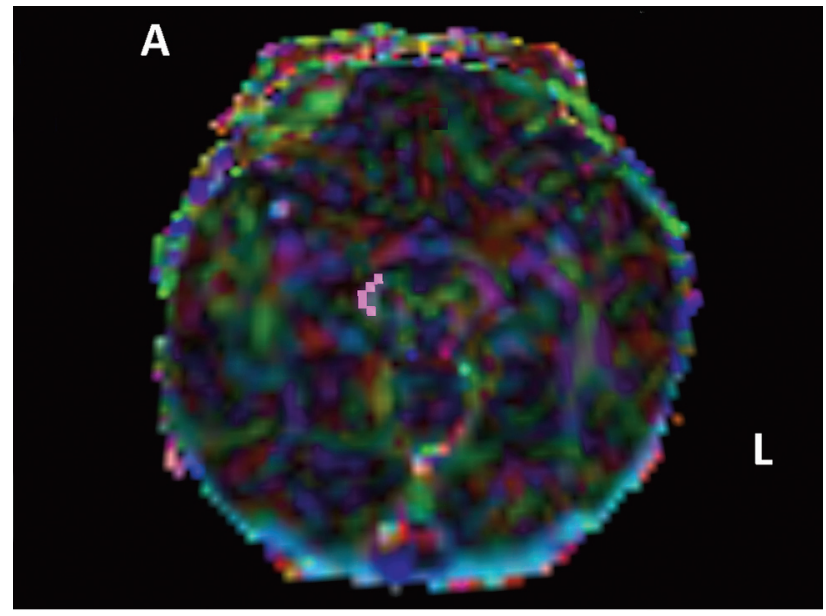

Fig 2. The CP region-of-interest positioning (purple squares) on native tensor diffusion map.

pressed by calculating the $\mathrm{CV}(\mathrm{CV}=\mathrm{SD} /$ mean $)$ after repeating measurements chosen at random, for 8 neonates. For intraobserver reproducibility, measurements were done 3 times for each neonate.

We performed group comparison using the Wilcoxon signedrank test for paired sample (comparison of left side vs the right side for each patient) and analysis of variance or the Kruskal-Wallis MultipleComparison Z-Value test for nonpaired data (Sarnat score vs MR imaging score, Sarnat score vs FA or ADC values) as appropriate. Comparison between quantitative data and early neurologic outcome was done with use of the Mann-Whitney nonparametric test. All statistical analyses were done on NCSS software (NCSS, Kaysville, Utah). Differences were considered significant if $P<.05$. For the KruskalWallis multiple-comparison Z-value test, differences in medians were considered significant if the $z$-value was more than 2.39 (Bonferroni correction).

\section{Results}

\section{Descriptive Clinical Data}

Complete analysis was performed in 22 neonates (on-line Table 1). The median Apgar score was 2 (22 infants) at birth and 5 (19 infants) at 5 minutes. Seven neonates had cardiac arrest at birth. Eight neonates required epinephrine. The Sarnat score was 3 in 13 children, 2 in 7 children, and 1 in 2 children. The median Sarnat score was 3. Thirteen neonates had seizures in the first 2 days of life; Amiel-Tison score was obtained for all live neonates and performed at $8.5 \pm 4$ days (median, 8 days). Group A included 16 infants ( 6 with moderate and 10 with no or minor neurologic abnormalities), and group B included 6 infants ( 5 deaths).

\section{Conventional MR Imaging and DWI Data Analysis}

All neonates underwent MR imaging in the first 6 days of life (median, 4 days; mean, $4 \pm 1.65$ days).

Descriptive analysis. Results are reported in on-line Tables 2 and 3. Lack of hyperintensity of the PLIC on T1WI (17 infants) and white matter abnormalities on DWI (15 infants) were the most often observed abnormalities. Brain swelling and petechial abnormalities of white matter (3 infants each) were unusual. Seven infants had brain stem abnormalities. Mean MR imaging score was $6.36 \pm 4.5$ (range, 1-18; median, 6).
Correlation between initial perinatal clinical examination and MR imaging score. We found no correlation between MR imaging score and Apgar score at 5 minutes or Sarnat score. Median MR imaging score was 4 in group A and 10 in group $\mathrm{B}(P=.02)$.

\section{DTI Data Analysis}

Descriptive analysis. Results are reported in on-line Table 4 (DTI data). Intraobserver comparison in FA and ADC measurements did not reveal statistical difference $(\mathrm{CV}<3 \%)$. For interobserver measurements, CV was less than $4 \%$ for the PLIC, less than $5 \%$ for the CP, and less than $4 \%$ for the PP. The median number of voxels on the left PLIC was 16 (range, 1224 ) and on the right PLIC was 18 (range, 11-33). The median number of voxels on the left PP was 80 (range, 46-110) and on the right PP was 76 (range, 48-101). Both for the left and the right side, the median number of voxels on the CP was 9 (left range, 4-15; right range, 5-14). Whatever the region of interest (ie, PLIC, PP, or CP), there was no significant difference between left and right FA or ADC values.

Correlation between perinatal clinical data and DTI measurements. The group with a poor Apgar score at 5 minutes had significant lower ADC values on PLIC and PP (right PLIC, $P=.05$; left PLIC, $P=.002$; right $\mathrm{PP}, P=.05$; left PP, $P=.01$ ) but not on CP. No statistical correlation was observed between the Sarnat score and DTI parameter measurements.

Correlation between DTI parameter measurements and early neurologic outcome. Results are summarized in the Table. FA was significantly decreased in group B vs group A only in the white matter areas (PLIC and CP) but not in PP whereas ADC was significantly decreased in group $B$ vs group $\mathrm{A}$ in PLIC and PP but not in CP.

\section{Discussion}

The main result of this pilot study is that DTI values measured in specific brain areas during the first 6 days of life correlated significantly with early neurologic outcome in infants with conditions likely to lead to HIE. With selective region-of-interest analysis, lower ADC values in PLIC and PP and lower FA values in PLIC and CP bilaterally correlated significantly with poor early neurologic outcome. These results suggest that DTI measurement could be efficient in the determination of early prognosis and that pathologic processes underlying adverse neurologic outcome are quite different in PLIC and PP compared with CP.

\section{Methodologic Considerations}

We reported clinical data and MR imaging parameters in a homogeneous cohort of 22 full-term neonates that fulfilled strict conditions with potential HIE. As ADC and FA values might change during the first weeks of life, specifically in $\mathrm{HI}$ conditions, the delay between the insult and MR imaging acquisition remains open to discussion. In our study, because of the risk for ADC pseudonormalization from the end of the first week, ${ }^{23}$ we decided to perform the MR imaging studies between day 1 and day 6 . We developed well-defined anatomic boundary points (identified separately in each patient) that depict the variation in DTI indices as a function of position within the brain. Manual region-of-interest selection allows measurements on native space, without image transforma- 


\begin{tabular}{|c|c|c|c|c|}
\hline \multicolumn{5}{|c|}{ Comparison between ADC and FA values on the left and right sides with early neurologic outcome } \\
\hline & & Group A & Group B & $P$ \\
\hline \multirow[t]{4}{*}{ PLIC } & Right ADC (mean [median] \pm SD) $\left(\mathrm{mm}^{2} / \mathrm{s}\right)$ & $1.101(1.115) \pm 0.071$ & $0.676(0.692) \pm 0.079$ & .000027 \\
\hline & Left ADC (mean [median] $\pm \mathrm{SD})\left(\mathrm{mm}^{2} / \mathrm{s}\right)$ & $1.074(1.082) \pm 0.068$ & $0.665(0.706) \pm 0.093$ & .000027 \\
\hline & Right FA (mean [median] \pm SD) & $0.461(0.458) \pm 0.057$ & $0.391(0.413) \pm 0.057$ & .006 \\
\hline & Left FA (mean [median] $\pm \mathrm{SD}$ ) & $0.457(0.455) \pm 0.047$ & $0.369(0.378) \pm 0.091$ & .02 \\
\hline \multirow[t]{4}{*}{ PP } & Right ADC (mean [median] \pm SD) & $1.177(1.162) \pm 0.077$ & $0.74(0.763) \pm 0.14$ & .000027 \\
\hline & Left ADC (mean [median] \pm SD) & $1.163(1.153) \pm 0.08$ & $0.722(0.749) \pm 0.14$ & .000027 \\
\hline & Right FA (mean [median] $\pm S D$ ) & $0.151(0.149) \pm 0.019$ & $0.148(0.134) \pm 0.32$ & .49 \\
\hline & Left FA (mean [median] $\pm S D$ ) & $0.155(0.150) \pm 0.021$ & $0.154(0.156) \pm 0.035$ & .74 \\
\hline \multirow[t]{4}{*}{$\mathrm{CP}$} & Right ADC (mean [median] \pm SD) & $1.391(1.369) \pm 0.265$ & $1.370(1.376) \pm 0.565$ & .91 \\
\hline & Left ADC (mean [median] $\pm S D$ ) & $1.4(1.367) \pm 0.26$ & $1.3(1.5) \pm 0.339$ & .85 \\
\hline & Right FA (mean [median] \pm SD) & $0.382(0.383) \pm 0.033$ & $0.312(0.316) \pm 0.058$ & .006 \\
\hline & Left FA (mean [median] $\pm S D$ ) & $0.385(0.381) \pm 0.043$ & $0.3(0.3) \pm 0.05$ & .003 \\
\hline
\end{tabular}

tion, and is more efficient for individual clinical analysis, whereas a voxel-based method requires normalization of data, which induces loss of anatomic precision. ${ }^{24}$ However, an a priori hypothesis concerning a few selected structures to be assessed is required. In our study, we selected PLIC and PP because of their known HI sensitivity. CP region of interest was chosen because 1) it mediates the communication of neuronal networks including telencephala, rhombencephala, and spinal cord (corticospinal tract); and 2) in adult cohorts, $\mathrm{CP}$ alteration has been described in patients with poor neurologic outcome in traumatic brain injury, in spinocerebellar ataxia types 1 and 2, and in stroke. ${ }^{25-27}$ In each case, anatomic landmarks for selected regions of interest were positioned according to Wakana et al. ${ }^{22}$ However, regions of interest drawn on DTI may have low resolution, but the high interrater and intrarater reliability demonstrates the reproducibility of the method.

\section{Early Neurologic Outcome and Imaging Parameters}

As previously shown, ${ }^{9,20,28}$ MR imaging score including conventional MR imaging and DWI data correlates significantly with early neurologic outcome of neonates with HIE conditions. The recent publication of Liauw et $\mathrm{al}^{28}$ supports the use of this type of score according to criterion standard injury patterns in HIE.

In our study, we showed that $\mathrm{ADC}$ values alone in the PP and PLIC, but not in CP, during the first days following HI brain injury appear as a sensitive marker to evaluate infants' early neurologic outcome as appreciated by the Amiel-Tison score. The lower the ADC value in those regions between day 1 and day 6 , the worse the early neurologic outcome. Our results are consistent with the literature. Hunt et $\mathrm{al}^{29}$ in 28 neonates with HIE found that the ADC value in the PLIC was significantly greater for those who survived compared with those who died. Barkovich et al (10 neonates with HIE) $)^{11}$ and Wolf et al ( 13 neonates with $\mathrm{HIE})^{30}$ reported an early high reduction of water diffusion in injured patients (up to $60 \%$ compared with control subjects) in specific brain areas (corticospinal tracts, thalami). Vermeulen et $\mathrm{al}^{31}$ confirmed the good prognostic value of early ADC measurement, particularly on the PLIC, to predict the prognosis of neonates with $\mathrm{HI}$ at 2 years. Some authors have suggested that an ADC value lower than $0.85 \times 10^{-3} \mathrm{~mm}^{2} / \mathrm{s}$ on PLIC is associated with a poor outcome. In our study, all of the neonates with a mean ADC value lower than $0.8 \times 10^{-3} \mathrm{~mm}^{2} / \mathrm{s}$ on the PLIC and $0.9 \times 10^{-3}$ $\mathrm{mm}^{2} / \mathrm{s}$ on the PP had a severe early neurologic outcome or died. Recently, Liauw et $\mathrm{al}^{32}$ in 24 neonates showed the strong interest of ADC measurement, particularly in regions with no visual abnormality on conventional MR imaging and DWI. The authors hypothesized that brain tissue may be disrupted despite normal MR imaging appearance, particularly in the basal ganglia and brain stem.

Bartha et $\mathrm{al}^{15}$ explored DTI data (ADC and FA) in white matter (optic radiation, corticospinal tracts, posterior white matter, frontal white mater) and gray matter (basal ganglia, thalami, calcarine cortex) of 14 healthy infants at term, giving reference values. However, FA measurement in a specific area in neonates with $\mathrm{HI}$ to predict outcome is poorly reported in the literature. Ward et $\mathrm{al}^{13}$ in 20 neonates with $\mathrm{HI}$ observed that FA remained decreased, whereas ADC pseudonormalized with time. At the same time, the authors reported that FA decreased in only some severe basal ganglia and white matter injury but decreased in all moderate basil ganglia and white matter injury. The authors' conclusion was that FA might represent an interesting marker in neonates, particularly in moderate $\mathrm{HI}$ and also when results of ADC values are normal. We could not observe such results in our study because we did not make chronologic comparisons in our predictive factors (ADC and FA).

Malik et $\mathrm{al}^{12}$ on 17 mild to moderate neonates with $\mathrm{HI}$ reported FA changes with time, showing an increase of this parameter between the 1st and 12th weeks of life. In our study, whereas FA values in PP did not correlate with early neurologic outcome, FA values in CP and PLIC areas did correlate with outcome, both on the right and left sides (ie, the lower the FA values in CP and PLIC, the worse the infants' early neurologic outcome). In our study, FA alteration on CP correlated with poor early neurologic outcome, whereas ADC alteration in the same area did not correlate with poor neurological outcome. In adult studies, a decrease of FA value on the CP in the first 2 weeks after ischemic stroke correlated with motor deficit at the time of imaging and 3 months later, whereas no pathologic findings have been observed with $\mathrm{T} 1$-weighted and T2-weighted images and ADC maps in these areas. ${ }^{26,27}$ These results in adult patients highlight the potentially strong interest in FA measurement regardng brain injury conditions, particularly on CP. Groenendaal et al ${ }^{33}$ explored the prewallerian degeneration of the $\mathrm{CP}$ in 39 neonates (term and near-term) with HI conditions. The authors showed the high positive predictive value of the association of PLIC and CP abnormal sig- 
nal intensity on DWI for delayed neurodevelopmental outcome. Moreover, in this study, 14 of the 15 neonates with prewallerian degeneration had a poor neurologic outcome. More recently, Domi et $\mathrm{al}^{34}$ reported the DWI changes in descending corticospinal tracts in CP in 14 neonates with acute arterial ischemic stroke. These authors showed that all children without signal intensity abnormality on descending corticospinal tracts had normal results on standardized neurologic examination after 1 year of life. FA and ADC values, imaging markers commonly used to study microstructural white or gray matter abnormalities, ${ }^{13,14}$ provide limited information for determination of specific pathologic processes at a cellular level. Moreover, the differential alteration in FA and ADC values we found here may reflect differences in pathologic processes, either in PLIC and PP or in CP areas. Low FA could reflect an early loss of structural integrity of fiber tracts that might explain our results on PLIC and CP, whereas ADC after an early phase of decrease in severe brain injury usually pseudonormalizes. Thus, the lowering of both FA and ADC in PLIC can reflect the severity of the injury in those infants. The hypothesis for only lower FA (not ADC) in CP in infants with a poor early neurologic outcome may be the occurrence of a prewallerian degeneration in the descending corticospinal tracts as has been suggested in neonatal and adult brain injury. ${ }^{26,33,34}$

Further studies are required to better understand physiopathologic processes leading to cerebral impairment in HIE. From a functional point of view, the DTI values probably represent part of the anatomic support behind the clinical perturbations observed in infants with HIE. Moreover, their correlation with early neurologic outcome suggests that these parameters could be robust prognostic factors for long-term outcome in infants with moderate disability.

\section{Limits of the Study}

Although encouraging, our results should be interpreted with caution because of the small size of our sample. For ethical reasons, there was no control group. In addition, region-ofinterest positioning may be difficult in some small areas, such as the CP. This finding invites us to be prudent in analyzing the results.

\section{Conclusions}

In our study, we showed that DTI parameters in specific brain areas had strong correlations with early neurologic outcome in infants with HIE, reflecting differences in damaged tissue mechanisms in the PLIC and PP or in the CP areas. Indeed, we can hypothesize that these indices could represent robust parameters to predict long-term outcome in HIE. Taking into account these results, studies are now needed 1) to evaluate the long-term prognostic value of DTI parameters (FA and ADC) in neonates with moderate injury; and 2) to discriminate among no disability, mild disability, and moderate disability. This could lead to the evaluation of protective treatments for a relatively short term.

\section{Acknowledgments}

The authors thank Prof. Chêne and Ms. S. Leproust from USMR of Bordeaux for their help with methodology, all of the pediatric radiologic unit workers, and the pediatric SMUR team of the University Hospital of Bordeaux. Also, the authors thank Philips for the opportunity to use PRIDE software for DTI data posttreatment, M. Bordessoules for excellent technical assistance, W. Mayo for helpful discussions, and Dr. L. Harper for his assistance with editing the manuscript in the English language. Part of this work was supported by $\mathrm{CHU}$ of Bordeaux (O.B.) and region of Aquitaine (M.A).

$\mathrm{O}$. Brissaud performed conception and design of the research, acquired, analyzed and interpreted the data, performed statistical analysis, handled funding and supervision, drafted the manuscript, and made critical revisions to the manuscript for important intellectual content. M. Amirault acquired, analyzed, and interpreted the data and performed statistical analysis. F. Villega acquired the data, handled funding and supervision, drafted the manuscript, and made critical revisions to the manuscript for important intellectual content. O. Periot acquired, analyzed, and interpreted the data and handled funding and supervision. J.F. Chateil handled conception and design of the research; acquired, analyzed, and interpreted the data; handled funding and supervision; drafted the manuscript; and made critical revisions to the manuscript for important intellectual content. M. Allard handled conception and design of the research, handled funding and supervision, drafted the manuscript, and made critical revisions to the manuscript for important intellectual content.

\section{References}

1. Wayenberg JL, Vermeylen D, Damis E. [Définition de l'asphyxie à la naissance et incidence des complications neurologiques et systémiques chez le nouveau-né à terme]. Arch Pediatr 1998;5:1065-71

2. Adamson SJ, Alessandri LM, Badawi N, et al. Predictors of neonatal encephalopathy in full-term infants. BMJ 1995;311:598-602

3. Ekert P, Perlman M, Steinlin M, et al. Predicting the outcome of postasphyxial hypoxic-ischemic encephalopathy within 4 hours of birth. J Pediatr 1997;131: 613-17

4. Finer NN, Robertson CM, Richards RT, et al. Hypoxic-ischemic encephalopathy in term neonates: Perinatal factors and outcome. J Pediatr 1981;98:112-17

5. Smith J, Wells L, Dodd K. The continuing fall in incidence of hypoxic-ischaemic encephalopathy in term infants. BJOG. 2000;107:461-66

6. Thornberg E, Thiringer K, Odeback, et al. Birth asphyxia: Incidence, clinica course and outcome in a Swedish population. Acta Paediatr 1995;84:927-32

7. Gosselin J, Gahagan S, Amiel-Tison C. The Amiel-Tison neurological assessment at term: conceptual and methodological continuity in the course of follow-up. Ment Retard Dev Disabil Res Rev 2005;11:34-51

8. Sarnat HB, Sarnat MS. Neonatal encephalopathy. Following foetal distress. A clinical and electroencephalography study. Arch Neurol 1976;33:696-705

9. Brissaud O, Chateil JF, Bordessoules M, et al. Chemical shift imaging and localised magnetic resonance spectroscopy in full-term asphyxiated neonates. Pediatr Radiol 2005;35:998-1005

10. Rutherford M, Counsell S, Allsop J, et al. Diffusion-weighted magnetic resonance imaging in term perinatal brain injury: a comparison with site of lesion and time from birth. Pediatrics 2004;114:1004-14

11. Barkovich AJ, Miller SP, Bartha A, et al. MR imaging, MR spectroscopy, and diffusion tensor imaging of sequential studies in neonates with encephalopathy. AJNR Am J Neuroradiol 2006;27:533-47

12. Malik GK, Trivedi R, Gupta RK, et al. Serial quantitative diffusion tensor MR of the term neonates with hypoxic-ischemic encephalopathy (HIE). Neuropediatrics 2006;37:337-43

13. Ward P, Counsell S, Allsop J, et al. Reduced fractional anisotropy on diffusion tensor magnetic resonance imaging after hypoxic-ischemic encephalopathy. Pediatrics 2006;117:e619-30

14. van Pul C, Buijs J, Janssen MJ, et al. Selecting the best index for following the temporal evolution of apparent diffusion coefficient and diffusion anisotropy after hypoxic-ischemic white matter injury in neonates. AJNR Am J Neuroradiol 2005;26:469-81

15. Bartha AI, Yap KR, Miller SP, et al. The normal neonatal brain: MR imaging, diffusion tensor imaging, and 3D MR spectroscopy in healthy term neonates. AJNR Am J Neuroradiol 2007;28:1015-21 
16. Cascio CJ, Gerig G, Piven J Diffusion tensor imaging: application to the study of the developing brain. J Am Acad Child Adolesc Psychiatry 2007;46:213-23

17. Hermoye L, Saint-Martin C, Cosnard G, et al. Pediatric diffusion tensor imaging: normal database and observation of the white matter maturation in early childhood. Neuroimage 2006;29:493-504

18. Miller SP, McQuillen PS, Hamrick S, et al. Abnormal brain development in newborns with congenital heart disease. N Engl J Med 2007;357:1928-38

19. Mukherjee P, Miller JH, Shimony JS, et al. Diffusion-tensor MR imaging of gray and white matter development during normal human brain maturation. AJNR Am J Neuroradiol 2002;23:1445-56

20. Meyer-Witte S, Brissaud O, Brun M, et al. [Apport pronostique de la resonance magnetique cerebrale dans l'encephalopathie hypoxique-ischemique du nouveau-ne a terme: Score d'imagerie, spectroscopie. Etude de 26 cas]. Arch Pediatr 2008; 15:9-23

21. Pajevic S, Pierpaoli C. Color schemes to represent the orientation of anisotropic tissues from diffusion tensor data: Application to white matter fiber tract mapping in the human brain. Magn Reson Med 1999;42:526-40

22. Wakana S, Jiang H, Nagae-Poetscher LM, et al. Fiber tract-based atlas of human white matter anatomy. Radiology 2004;230:77-87

23. Winter JD, Lee DS, Hung RM, et al. Apparent diffusion coefficient pseudonormalization time in neonatal hypoxic-ischemic encephalopathy. Pediatr Neurol 2007;37:255-62

24. Snook L, Plewes C, Beaulieu C. Voxel based versus region of interest analysis in diffusion tensor imaging of neurodevelopment. Neuroimage 2007;34:243-52

25. Mandelli ML, De Simone T, Minati L, et al. Diffusion tensor imaging of spinocerebellar ataxias types 1 and 2. AJNR Am J Neuroradiol 2007;28:1996-2000

26. Sidaros A, Engberg AW, Sidaros K, et al. Diffusion tensor imaging during recovery from severe traumatic brain injury and relation to clinical outcome: a longitudinal study. Brain 2008;131:559-72

27. Thomalla G, Glauche V, Koch MA, et al. Diffusion tensor imaging detects early wallerian degeneration of the pyramidal tract after ischemic stroke. Neuroimage 2004;22:1767-74

28. Liauw L, van der Grond J, van den Berg-Huysmans AA, et al. Hypoxic-ischemic encephalopathy: diagnostic value of conventional MR imaging pulse sequences in term-born neonates. Radiology 2008;247:204-12

29. Hunt RW, Neil JJ, Coleman LT, et al. Apparent diffusion coefficient in the posterior limb of the internal capsule predicts outcome after perinatal asphyxia. Pediatrics 2004;114:999-1003

30. Wolf RL, Zimmerman RA, Clancy R, et al. Quantitative apparent diffusion coefficient measurements in term neonates for early detection of hypoxicischemic brain injury: Initial experience. Radiology 2001;218:825-33

31. Vermeulen RJ, van Schie PE, Hendrikx L, et al. Diffusion-weighted and conventional MR imaging in neonatal hypoxic ischemia: two-year follow-up study. Radiology 2008;249:631-39

32. Liauw L, van Wezel-Meijler G, Veen S, et al. Do apparent diffusion coefficien measurements predict outcome in children with neonatal hypoxic-ischemic encephalopathy? AJNR Am J Neuroradiol 2009;30:264-70

33. Groenendaal F, Benders MJ, de Vries LS. Pre-wallerian degeneration in the neonatal brain following perinatal cerebral hypoxia-ischemia demonstrated with MRI. Semin Perinatol 2006;30:146-50

34. Domi T, deVeber G, Shroff $\mathrm{M}$, et al. Corticospinal tract pre-wallerian degeneration: a novel outcome predictor for pediatric stroke on acute MRI. Stroke 2009;40:780-87 